\title{
8-DIMENSIONAL EINSTEIN-THORPE MANIFOLDS
}

\author{
JAEMAN KIM
}

(Received 21 April 1999; revised 21 September 1999)

\author{
Communicated by K. Ecker
}

\begin{abstract}
We prove that a compact orientable Einstein-Thorpe manifold of dimension 8 that satisfies $6 \chi=\left|P_{2}\right|$ must be flat.

1991 Mathematics subject classification (Amer. Math. Soc.): primary 53B20, 53B21, 53C25.

Keywords and phrases: Einstein-Thorpe manifold, flat.
\end{abstract}

\section{Introduction}

The local geometry of a manifold provides us with information about its global topology. For instance, the generalized Gauss-Bonnet theorem [2, 5] states that the Euler-Poincaré characteristic $\chi$ of a compact oriented Riemannian manifold $M^{4 k}$ can be written as an integral

$$
\chi=\frac{2}{V} \frac{[(2 k) !]^{2}}{4 k} \int_{M} \operatorname{trace}\left(* R_{2 k} * R_{2 k}\right) d V,
$$

where $V$ is the volume of the Euclidean unit $4 k$-sphere, $d V$ is the volume element of $\mathrm{M}, *$ is the Hodge $*$-operator, and $R_{2 k}$ is called the $2 k$ th curvature operator. If $R_{2 k}$ commutes with $*$, that is, $R_{2 k} *=* R_{2 k}$, we call this condition a Thorpe condition and this metric a Thorpe metric and this manifold a Thorpe manifold. If Ricci curvature, ric, is a constant multiple of the metric, equivalently, traceless Ricci curvature, ric , $_{0}$ vanishes then we call this condition an Einstein condition and this metric an Einstein metric and this manifold an Einstein manifold. In the 4-dimensional case, the Thorpe condition is equivalent to the Einstein condition [1]. And so, in 4 dimensions there is another way of stating the Einstein equation, namely the Thorpe condition. Moreover,

(c) 2000 Australian Mathematical Society $0263-6115 / 2000 \$ A 2.00+0.00$ 
in case of a compact oriented Einstein manifolds of dimension 4 with $2 \chi=\left|P_{1}\right|$, Hitchin in [3] has classified these manifolds. On the other hand, Thorpe metrics need not be Einstein in dimensions higher than 4 and the following metrics satisfy the Thorpe condition but they are not Einstein metrics [4]:

(i) $S^{4 k} \times H^{4 k}$, product metric of standard metrics;

(ii) $C P^{2} \times C H^{2}$, product metric of standard metrics.

The following examples also provide us with Einstein manifolds but not Thorpe manifolds [4]: the canonical quaternion projective space $H P^{n}$ with $n \geq 3$.

We say that a Riemannian $4 k$ manifold is Einstein-Thorpe if it is both Einstein and Thorpe. The purpose of the present note is to see what happens to Hitchin's result if both conditions are imposed in dimension eight.

THEOREM 1.1. Suppose that $\left(M^{8}, g\right)$ is a compact orientable Einstein-Thorpe manifold and that

$$
\chi=\left(\frac{2 ! 2 !}{4 !}\right)\left|P_{2}\right|
$$

Then $\left(M^{8}, g\right)$ must be a flat manifold.

The crucial ingredient in the proof is Lemma 1.1.

LEMMA 1.1. Let $(M, g)$ be a Riemannian manifold of dimension 8 . Then

$$
\operatorname{trace} R_{4}=\frac{1}{2^{2}}\left(\frac{1}{6}\right)\left\{\frac{1}{2} S^{2}-4 \mid \text { ric }\left._{0}\right|^{2}+4|R|^{2}\right\}
$$

where $S$ is the scalar curvature, ric $_{0}$ is the traceless Ricci curvature and $R$ is the curvature.

From Lemma 1.1 we can observe that trace $R_{4}$ is nonnegative when the Riemannian manifold is Einstein.

\section{The $p$ th curvature operator and Thorpe manifolds}

Let $M$ be a Riemannian manifold of dimension $n$ and let $\wedge^{p}(M)$ denote the bundle of $p$-vectors of $M ; \wedge^{p}(M)$ is a Riemannian vector bundle, with inner product on the fiber $\bigwedge^{p}(x)$ over the point $x$ [4]. Let $R$ denote the covariant curvature tensor of $M$. For each even $p>0$, we define the $p$ th curvature tensor $R_{p}$ of $M$ to be the covariant 
tensor field of order $2 p$ given by

$$
\begin{aligned}
& R_{p}\left(u_{1}, \ldots, u_{p}, v_{1}, \ldots, v_{p}\right) \\
& =\frac{1}{2^{p / 2} p !} \sum_{\alpha, \beta \in S_{p}} \varepsilon(\alpha) \varepsilon(\beta) R\left(u_{\alpha(1)}, u_{\alpha(2)}, v_{\beta(1)}, v_{\beta(2)}\right) \ldots \\
& \quad R\left(u_{\alpha(p-1)}, u_{\alpha(p)}, v_{\beta(p-1)}, v_{\beta(p)}\right),
\end{aligned}
$$

where $u_{i}, v_{j} \in T_{x} M$ and $S_{p}$ denotes the group of permutations of $(1, \ldots, p)$ and, for $\alpha \in S_{p}, \varepsilon(\alpha)$ is the sign of the permutation $\alpha$.

The tensor $R_{p}$ has the following properties: it is alternating in the first $p$ variables, alternating in the last $p$ variables and it is invariant under the operation of interchanging the first $p$ variables with the last $p$ variables. Hence, at each point $x \in M, R_{p}$ can be regarded as a symmetric bilinear form on $\bigwedge^{p}(x)$. By use of the inner product on $\bigwedge^{p}(x), R_{p}$ at $x$ may then be identified with a self-adjoint linear operator $R_{p}$ on $\bigwedge^{p}(x)$. Explicitly, this identification is given by

$$
\left\langle R_{p}\left(u_{1} \wedge \cdots \wedge u_{p}\right), v_{1} \wedge \cdots \wedge v_{p}\right\rangle \equiv R_{p}\left(u_{1}, \ldots, u_{p}, v_{1}, \ldots, v_{p}\right)
$$

with $u_{i}, v_{j} \in T_{x} M$. From now on, we use the same notations for the $p$ th curvature operators and the $p$ th curvature tensors. The tensor $R_{p}$ satisfies the Bianchi identity which can be expressed in the following way [5]:

$$
\text { Alt } R_{p}=0 \text {, }
$$

where Alt is the skew symmetrization operator given by

$$
\text { Alt } R_{p}\left(v_{1}, \ldots, v_{2 p}\right)=\frac{1}{(2 p) !} \sum_{r \in S_{2 p}} \varepsilon(r) R_{p}\left(v_{r(1)}, \ldots, v_{r(2 p)}\right)
$$

with $v_{i} \in T_{x} M$.

When $n$ is a multiple of $4, p=n / 2$ and $M$ is oriented, the Bianchi identity for $R_{p}$ admits another interpretation in terms of the Hodge star operator on $\bigwedge^{p}(M)$ :

$$
\text { Alt } R_{p}\left(e_{1}, \ldots, e_{n}\right)=\frac{p ! p !}{n !} \text { trace } * R_{p}
$$

and hence for the case $p=n / 2$, the Bianchi identity for $R_{p}$ reduces to

$$
\text { trace } * R_{p}=0 \text {. }
$$

Taking $p=n$, the space $\bigwedge^{n}(x)$ is one dimensional and hence the self-adjoint linear operator $R_{n}: \bigwedge^{n}(x) \rightarrow \bigwedge^{n}(x)$ is a scalar multiple of the identity. More explicitly, when expressed globally, the line bundle homomorphism $R_{n}: \bigwedge^{n}(M) \rightarrow \bigwedge^{n}(M)$ is

$$
R_{n}=K I
$$


where $I$ is the identity automorphism of $\bigwedge^{n}(M)$ and $K$ is the Lipschitz-Killing curvature of $M$. Furthermore, for $x \in M$,

$$
K(x)=R_{n}\left(e_{1}, \ldots, e_{n}, e_{1}, \ldots, e_{n}\right),
$$

where $\left\{e_{1}, \ldots, e_{n}\right\}$ is any orthonormal basis for $T_{x} M$. The generalized Gauss-Bonnet theorem [5] expresses the Euler-Poincaré characteristic $\chi$ of a compact oriented Riemannian manifold of even dimension $n$ as an integral

$$
\chi=\frac{2}{c_{n}} \int_{M} K d V,
$$

where $K$ is the Lipschitz-Killing curvature of $M, c_{n}$ is the volume of Euclidean unit $n$-sphere and $d V$ is the volume element of $M$. Now we show that the Lipschitz-Killing curvature $K$ of $M$ can be expressed in terms of $R_{p}$ and the Hodge *-operator. Let $M$ be an oriented Riemannian manifold of even dimension $n$, then according to [5], the Lipschitz-Killing curvature $K$ of $M$ is the function whose value at $x \in M$ is

$$
\frac{p !(n-p) !}{n !} \operatorname{trace}\left(* R_{n-p} * R_{p}\right) \text {. }
$$

For an oriented Riemannian manifold of dimension $n=4 k$, we can consider the middle curvature operator $R_{2 k}$, and if this operator satisfies the condition

$$
R_{2 k} *=* R_{2 k},
$$

then, since $*^{2}=$ Identity, the trace formula for $K$ reduces to

$$
K=\frac{[(2 k) !]^{2}}{(4 k) !} \operatorname{trace} R_{2 k}^{2} \geq 0 .
$$

Next we consider a necessary condition for the existence of a Thorpe metric [5]:

THEOREM 2.1. Let $M$ be a compact orientable $4 k$-dimensional Riemannian manifold which admits a Thorpe metric. Then

$$
\chi \geq \frac{k ! k !}{(2 k) !}\left|P_{k}\right|
$$

where $\chi$ is the Euler characteristic of $M$ and $P_{k}$ is the kth Pontrjagin number of $M$. In particular, $\chi \geq 0$. Furthermore, $\chi=0$ if and only if $R_{2 k}=0$.

PROOF. The de Rham representation for the $k$ th Pontrjagin class of $M$ [2] is the differential $4 k$-form

$$
\frac{[(2 k) !]^{3}}{\left(2^{k} k !\right)^{2}(2 \pi)^{2 k}} \operatorname{trace}\left(R_{2 k} * R_{2 k}\right) d V
$$


Since $R_{2 k}$ commutes with $*$ it also commutes with $I \pm *$, where $I$ denotes the identity operator on $\bigwedge^{2 k}$. Hence $R_{2 k}(I \pm *)$ is self-adjoint and

$$
0 \leq \operatorname{trace}\left[R_{2 k}(I \pm *)\right]^{2}=2\left[\operatorname{trace}\left(R_{2 k}\right)^{2} \pm \operatorname{trace}\left(R_{2 k} * R_{2 k}\right)\right],
$$

and so

$$
\operatorname{trace}\left(R_{2 k}\right)^{2} \geq\left|\operatorname{trace}\left(R_{2 k} * R_{2 k}\right)\right|
$$

This means that

$$
\chi \geq \frac{k ! k !}{(2 k) !}\left|P_{k}\right|
$$

and since $K \geq 0$, we have $\chi=0$ if and only if $K$ is identically zero. $K \equiv 0$ is equivalent to $R_{2 k}=0$ and this completes the proof.

\section{The case of $\chi=((2 ! 2 !) / 4 !)\left|P_{2}\right|$}

In this section we prove that a compact orientable Einstein-Thorpe manifold of dimension 8 that satisfies the above topological equality must be flat.

LEMMA 3.1. Let $(M, g)$ be a Riemannian manifold of dimension 8. Then

$$
\operatorname{trace} R_{4}=\frac{1}{2^{2}}\left(\frac{1}{6}\right)\left\{\frac{1}{2} S^{2}-4 \mid \text { ric }\left._{0}\right|^{2}+4|R|^{2}\right\}
$$

where $S$ is the scalar curvature, ric ${ }_{0}$ is the traceless Ricci curvature and $R$ is the curvature.

PROOF. For 4-forms $\left\{e_{a} \wedge e_{b} \wedge e_{c} \wedge e_{d}\right\}$ and with the Einstein summation,

$$
\begin{aligned}
\operatorname{trace} R_{4} & =\frac{1}{2^{2}} R_{[a b}^{[a b} R_{c d]}^{c d]}=\frac{1}{2^{2}} R_{[a b}^{a b} R_{c d]}^{c d} \\
& =\frac{1}{2^{2}}\left(\frac{1}{6}\right)\left\{R_{a b}^{a b} R_{c d}^{c d}+R_{a c}^{a b} R_{d b}^{c d}+R_{a d}^{a b} R_{b c}^{c d}+R_{b c}^{a b} R_{a d}^{c d}+R_{b d}^{a b} R_{c a}^{c d}+R_{c d}^{a b} R_{a b}^{c d}\right\}
\end{aligned}
$$

where [ ] is a skew symmetrization, and $\left\{e_{k}\right\}_{k=1}^{8}$ is an orthonormal frame. We analyze the terms of this sum individually:

(i) $R_{a b}^{a b} R_{c d}^{c d}=1 / 2 S^{2}-4 \mathrm{ric}_{0 c}^{c} \mathrm{ric}_{0 c}^{c}+2 R_{c d}^{c d} R_{c d}^{c d}$;

(ii) $R_{a c}^{a b} R_{d b}^{c d}=-\mathrm{ric}_{0 c}^{b} \mathrm{ric}_{0 b}^{c}+R_{d c}^{d b} R_{d b}^{d c}$. 
Thus we obtain

$\operatorname{trace} R_{4}=\frac{1}{2^{2}}\left(\frac{1}{6}\right)\left\{\frac{1}{2} S^{2}-4 \mathrm{ric}_{0 c}^{c} \operatorname{ric}_{0 c}^{c}-4 \mathrm{ric}_{0 c}^{b} \operatorname{ric}_{0 b}^{c}+2 R_{c d}^{c d} R_{c d}^{c d}+4 R_{d c}^{d b} R_{d b}^{d c}+R_{c d}^{a b} R_{a b}^{c d}\right\}$ and this completes the proof.

Now we are ready to prove the main result.

THEOREM 3.1. Suppose that $\left(M^{8}, g\right)$ is a compact orientable Einstein-Thorpe manifold and that

$$
\chi=\left(\frac{2 ! 2 !}{4 !}\right)\left|P_{2}\right|
$$

Then $\left(M^{8}, g\right)$ must be a flat manifold.

PROOF. By Theorem 2.1, we see that the above topological condition together with the Thorpe condition can be expressed as

$$
\text { trace } R_{4} R_{4}=\mid \text { trace } R_{4} * R_{4} \mid \text {. }
$$

We consider any orthonormal basis $\left\{A_{i}\right\}_{i=1}^{14}$ in $\bigwedge^{+}\left(M^{8}\right)$, and any orthonormal basis $\left\{B_{i}\right\}_{i=1}^{14}$ in $\bigwedge^{-}\left(M^{8}\right)$, where $\bigwedge^{+}\left(M^{8}\right)$ and $\bigwedge^{-}\left(M^{8}\right)$ denote the self dual space and the anti-self-dual space with respect to the Hodge $*$ operator, respectively. Then we have

$$
\begin{aligned}
\left\langle R_{4}\left(A_{i}\right), R_{4}\left(A_{i}\right)\right\rangle & =\sum_{j=1}^{14}\left|R_{4}\left(A_{i}, A_{j}\right)\right|^{2}+\sum_{j=1}^{14}\left|R_{4}\left(A_{i}, B_{j}\right)\right|^{2}, \\
\left\langle R_{4}\left(B_{i}\right), R_{4}\left(B_{i}\right)\right\rangle & =\sum_{j=1}^{14}\left|R_{4}\left(B_{i}, B_{j}\right)\right|^{2}+\sum_{j=1}^{14}\left|R_{4}\left(B_{i}, A_{j}\right)\right|^{2}, \\
\left\langle * R_{4}\left(A_{i}\right), R_{4}\left(A_{i}\right)\right\rangle & =\sum_{j=1}^{14}\left|R_{4}\left(A_{i}, A_{j}\right)\right|^{2}-\sum_{j=1}^{14}\left|R_{4}\left(A_{i}, B_{j}\right)\right|^{2}, \\
\left\langle * R_{4}\left(B_{i}\right), R_{4}\left(B_{i}\right)\right\rangle & =\sum_{j=1}^{14}\left|R_{4}\left(B_{i}, A_{j}\right)\right|^{2}-\sum_{j=1}^{14}\left|R_{4}\left(B_{i}, B_{j}\right)\right|^{2},
\end{aligned}
$$

for each $i=1,2, \ldots, 14$.

If we assume trace $R_{4} * R_{4} \geq 0$, then by the given condition

$$
R_{4}\left(A_{i}, B_{j}\right)=R_{4}\left(B_{i}, B_{j}\right)=0 \quad \text { for } i, j=1,2, \ldots, 14
$$

and this means that

$$
R_{4}^{-}=\frac{R_{4}-* R_{4}}{2} \equiv 0
$$


Furthermore, by the Bianchi identity,

$$
\text { trace } * R_{4} \equiv 0,
$$

and so we obtain

$$
\text { trace } R_{4} \equiv 0 \text {. }
$$

However, by Lemma 3.1 the Einstein condition (that means ric $c_{0}=0$ ) implies trace $R_{4} \geq 0$ and equality holds when its metric is flat and so we conclude that the given metric is flat.

On the other hand, if we assume

$$
\text { trace } R_{4} * R_{4} \leq 0 \text {, }
$$

then we can repeat the above argument with a different choice of sign and this completes the proof.

COROLLARY 3.1. (i) The product manifold of $T^{4}$ with any compact orientable hyperbolic manifold of dimension 4 does not admit an Einstein-Thorpe metric.

(ii) The product manifold of $T^{4}$ with any compact complex hyperbolic manifold of complex dimension 2 does not admit an Einstein-Thorpe metric.

The manifolds described in (i) and (ii) satisfy $\chi=0$ and $P_{2}=0$.

PROOF. It is easy to see that the manifolds described in part (i) and (ii) satisfy $\chi=0$ and $P_{2}=0$. This implies that any Einstein-Thorpe metric on the manifolds described in (i) and (ii) must be flat by Theorem 3.1, hence a contradiction.

\section{References}

[1] A. Besse, Einstein manifolds (Springer, Berlin, 1986).

[2] S. S. Chern, 'A simple intrinsic proof of the Gauss-Bonnet theorem for closed Riemannian manifolds', Ann. of Math. 45 (1994), 747-752.

[3] N. J. Hitchin, 'Compact four-dimensional Einstein manifolds', J. Differential Geom. 9 (1974), $435-444$.

[4] J. M. Kim, Einstein-Thorpe manifolds (Ph.D. Thesis, S.U.N.Y at Stony Brook, 1998).

[5] J. A. Thorpe, 'Some remarks on the Gauss-Bonnet integral', J. Math. Mech. 18 (1969), 779-786.

Department of Mathematics

Yonsei University

Shinchon 134

Seoul

Korea

e-mail: jaeman@math.yonsei.ac.kr 